\title{
INNOVACIÓN PARA LA GESTIÓN DE TECNOLOGÍA SOCIAL (TS) EN EL CAMPO DEL HÁBITAT. Experiencias de Desarrollo de Tec- nología Social Co-construida en San Carlos de Bariloche.
}

Santiago Ríos; Valeria Fenoglio; Josefina Centeno; Ana Laura Minari; Rodrigo Fabre; Paula Peyloubet

Programa de Estudio: "Co-construcción del conocimiento".

Centro de Investigaciones y Estudio sobre Cultura y Sociedad - CIECS-CONICET-UNC.

Valparaiso $\mathrm{s} / \mathrm{n}^{\circ}$. Ciudad Universitaria - Córdoba. Argentina.

03515353769 (interno 77011 )

Email de contacto: arqsantirios@gmail.com; paulapeyloubet@hotmail.com

Palabras clave: tecnología social, producto, proceso, gestión, co-construcción del conocimiento.

\section{Resumen:}

El presente trabajo se basa en el desarrollo de estrategias para la innovación tecnológica, a partir de procesos asociativos y redes de investigación multidisciplinarias, articulados con los contextos socio-productivos locales más vulnerables, promoviendo una efectiva participación ciudadana en la gestión pública. Las experiencias que se presentan en este trabajo, abordan la gestión tecnológica, el desarrollo tecnológico (producto) y la producción tecnológica (proceso) en el marco de un grupo de actores productivos y políticos involucrados y sus contextos para generar nuevas lógicas de gestión, que procuran propiciar procesos de articulación interactoral e intersectorial a partir de una gestión asociada público-privada.

El concepto de Tecnología Social (TS), articula tres niveles distinguibles (sólo a nivel analítico porque en la práctica van íntimamente unidos): producto, proceso y gestión; donde el producto advierte sobre lo artefactual-objeto arquitectónico, el proceso refiere a la trama de relaciones interactorales en las que se produce conocimiento y se construye, y por último la gestión que aborda las interrelaciones que se generan entre las instituciones y sus ideologías para la toma de decisiones.

La experiencia que se presenta en este trabajo, corresponde a un caso que se encuentra en desarrollo en la actualidad en la ciudad de San Carlos de Bariloche. En ella puede reconocerse aspectos generales tales como: el desarrollo de tecnología social en el campo del Hábitat, la generación de redes socio-productivas, la co-construcción de conocimientos, y aspectos particulares en relación al territorio, sus condiciones materiales, sus recursos humanos y aún en las experiencias sensibles que cada participante evoca a partir de los encuentros.

\section{Introducción.}

La problemática de la pobreza y la exclusión de la dinámica económica y el potencial crecimiento material de grandes sectores de la población, son problemas aún no resueltos en nuestro país y la región. Se deben reconocer los aspectos que harían posible la transformación de esta situación planteada, de pobreza y exclusión, teniendo en cuenta abordajes diferenciados a partir de la recreación-revisión de sistemas de producción tecnológica basados en cooperación, solidaridad y redistribución de bienes-riquezas y recursos, procurando revertir algunos conceptos de la economía formal-paradigma capitalista actual- hacia una economía social -nuevo paradigma solidario- abandonando la lógica de producción convencional excluyente.

El desarrollo tecnológico, orientado hacia la resolución de problemas sociales bajo una lógica solidaria, reviste una importancia estratégica para nuestra región. La inclusión de comunidades y grupos sociales dependerá de la capacidad local de genera soluciones tecnológicas y productivas incluyentes.

En este contexto es que se vislumbran dos ejes posibles para abordar el desarrollo tecnológico inclusivo. Uno, se refiere a la incorporación del saber popular al sustrato cognitivo de resolución 
de problemas tecnológicos. Otro, se refiere a la adecuación de las tecnologías y sus modalidades productivas a partir de lógicas solidarias y cooperativas, para consolidar nuevos modelos de desarrollo.

El problema queda planteado entonces: la inexistencia de un modelo de desarrollo incluyente gravita sobre la falta de participación de los actores afectados en la resolución del problema, co-construcción tecnológica, y la falta de mecanismos adecuados para poner en marcha las nuevas tecnologías. A partir de esta propuesta se sostiene el concepto de Tecnología Social (TS) (Dagnino, 2004).

\section{Desarrollo conceptual.}

"La pluralidad de opinión es necesaria para el conocimiento [...] y un método que fomente la pluralidad es el único método compatible con una perspectiva humanista [...] el conocimiento se obtiene de una proliferación de puntos de vista más que una aplicación determinada de la ideología preferida [...] El primer paso del camino hacia una cosmología nueva [...] es un paso hacia atrás [...] Sin caos, no hay conocimiento. Sin un olvido frecuente de la razón, no hay progreso. Las ideas que hoy día constituyen la base misma de la ciencia existen sólo porque hubo cosas tales como el prejuicio, el engaño y la pasión; porque estas cosas se opusieron a la razón; y porque se les permitió seguir su camino" (Paul Feyerabend, 2010: 29-166).

Los modelos de desarrollo imperantes en el sistema económico mundial, se basan en el juego de oferta y demanda en donde el libre mercado, de servicios y productos, se autorregula para dar lugar a crecimientos inequitativos y decrecimientos fabulosos a la vez, que no aseguran la capacidad de subsistencia de muchos sectores, poniendo en riesgo los procesos de democratización y gobernabilidad.

La Tecnología (T), entendida como producto-proceso-gestión (Peyloubet, 2017), no es neutra. Sin lugar a dudas posee ideología y metas que responden a determinadas maneras de pensar los modelos de desarrollo posibles, para qué y para quiénes.

Según Dagnino (2011), existen Tecnologías Convencionales (TC) que generan, en el modelo vigente, juegos de mercadotecnia de los que se benefician unos pocos y se excluyen muchos. Esta lógica de TC es sin duda el aparato montado que beneficia a ciertos sectores económicos que detentan el poder y forman parte del sector de relevancia política y decisoria.

En las décadas del ' 60 y '70 surgen nuevas reflexiones acerca de la tecnología. Los ideales de justicia e inclusión penetran en el espacio del desarrollo tecnológico, procurando colocar este juego de oferta y demanda al alcance de toda la población. Surge por aquella época el concepto de Tecnología Apropiada (TA) que intenta sumar la población excluida a la lógica de mercado y de renta de la TC. Los esfuerzos y buena voluntad por reclamar un espacio en este escenario permitieron poner en marcha, con cortos alcances, una visión integral de necesidades y demandas de los sectores pobres de la sociedad. Sin embargo, el gran equívoco en ese momento fue que el "juego de oferta y demanda" se instalaron con un nuevo nombre consagrando, dentro de la TA, a la Transferencia Tecnológica (TT) que marcó una brecha entre expertos y capacitados que relegó nuevamente al sector pobre al campo de los excluidos.

La TT, de la mano de la TA, trajo consigo la idea de que las necesidades de los sectores pobres y excluidos podían ser resueltas por el sector líder de producción cognitiva (sector de CyT) y ofrecidas "generosamente" para su uso. En ese camino se desestimó el propio sector beneficiario como potencial colaborador de la resolución del problema y la producción sectorizada invalidó la respuesta con el tiempo. Los problemas sociales, como resultado de este proceso, tendieron a ser definidos a partir de modelos cognitivos de otros actores sociales y no a partir de la visión de sector que poseía el problema. El fracaso dio lugar a la idea de que los problemas no podían ser postulados a priori ni a partir de la sola intervención de los sectores no afectados. A partir de 
estas posiciones se comienzan a gestar nuevas ideas que relacionan al desarrollo tecnológico con el desarrollo social, insumos para una inclusión social real que dan lugar a la TS.

Así fue sustituida la ingenua e ineficaz idea de "oferta y demanda" ○ "transferencia tecnológica", producida por la comunidad de expertos, por una construcción colectiva de conocimiento (co-construcción) que incorpora los valores, intereses, necesidades y saberes de los sectores excluidos.

Pero el problema planteado en este campo, no reside únicamente en la falta de incorporación al sustrato cognitivo de resolución de un problema el saber popular sino que, otro grave problema detectado y aún no considerado, es la inadecuación de las tecnologías actuales, concebidas éstas en la coherencia de los valores y necesidades empresariales que responden a una lógica mercantil, que nada tienen que ver con las lógicas solidarias y cooperativas que necesitan los nuevos modelos de desarrollo incluyentes.

No se agota entonces el problema con la propuesta de participación interactoral en la construcción del conocimiento y en la definición tecnológica, sino que se hace imprescindible el cambio de estrategias que operen sobre las relaciones de poder y decisión para que la tecnología realmente pueda generar beneficios para toda las personas (Thomas, 2009).

En síntesis, la inexistencia de un modelo de desarrollo verdaderamente incluyente gravita sobre la falta de participación de los actores afectados en la resolución del problema, co-construcción o construcción colectiva de la tecnología, y la falta de mecanismos adecuados para poner en marcha las nuevas ideas de desarrollo tecnológico, que no pueden echarse a andar sobre la lógica de TC, de visión empresarial y mercantil, o de la TA, de visión reduccionista y algo filantrópica, sino que deben construirse sobre la base de nuevas formas de hacer, pensar y sentir dando lugar a una efectiva participación democrática en el desarrollo de la tecnología.

\section{Desarrollo de la experiencia.}

La experiencia que se comparte en este trabajo se viene desarrollando desde el año 2013 en la ciudad de San Carlos de Bariloche y apunta a un desarrollo de tecnología para construcción en madera (impulsando el uso sustentable del recurso regional) en el marco de circuitos productivos interactorales que promuevan la diversificación de la economía local, apalancando los sectores más vulnerables de la comunidad.

Se trata de un trabajo de investigación-acción que asienta su trayectoria en dos metas fundamentales, inherentes al Plan de Ciencia y Tecnología (2014): "Argentina Innovadora 2020".

La primera se refiere al abordaje de temas estratégicos del país, que en estas experiencias se posiciona específicamente sobre la temática del Desarrollo de Tecnología Social. La segunda, se refiere al aprovechamiento de los potenciales productivos territoriales de cada región que se asientan sobre recursos renovables y sustentables. En este caso se hace referencia a la producción forestal. Se deja por supuesto que el uso de especies maderables no convencionales para su aplicación en la construcción de vivienda y el desarrollo de tecnología innovadora, generará valor agregado a la producción forestal, propiciando entramados productivos diversificados con capacidad de generar trabajo.

En el marco de estas metas generales se inserta esta experiencia que pretende reconocer la potencialidad de un material poco valorado, como es el pino ponderosa, como insumo protagónico en la construcción, tanto de vivienda nueva como de componentes para mejoramiento habitacional, procurando traccionar la cadena de valor de este sector productivo relegado en la zona.

La estrategia de implementación se basa en una innovación tecnológica, a partir de procesos asociativos y redes interactorales, que promuevan una directa articulación con los contextos socio-productivos más vulnerables y la gestión pública. 
La propuesta se inicia con el reconocimiento de un problema generado a partir de un ineficiente uso de un recurso natural renovable -madera de pino ponderosa. A partir de este reconocimiento se implementa la idea de diversificar el uso del recurso, basándose en la producción de componentes para la construcción (viviendas y edificios públicos), situación que responde sinérgicamente a la demanda del déficit habitacional y la necesidad de empleo sostenible y trabajo autónomo de la mencionada localidad.

Se reconocen en esta experiencia tres componentes sistémicos del problema mencionado.

El primero, sistema productivo forestal, que refiere a los sistemas de producción en donde se observa un escaso grado de ejecución de prácticas silvícolas, por la falta de salida comercial para los rollizos, provocando una inviabilidad económica en la producción y, a su vez, una pérdida del valor de las plantaciones. La implantación forestal no ha alcanzado el ritmo deseado para el crecimiento del sector. La dependencia exclusiva del sistema de promoción nacional dejó supeditado al mismo a los vaivenes económicos del Estado nacional y sus políticas intermitentes. La inmovilización del capital, por los tiempos de corta, ha ocasionado que las inversiones privadas se realicen en otras actividades económicas. Los programas de promoción fueron ineficientes para generar mecanismos atractivos a los distintos actores involucrados de la cadena foresto-industrial. Por otro lado, la baja producción de pino ponderosa -resultado de su escasa silvicultura- genera en la localidad y la región circuitos productivos cortos -leña- que no permiten el agregado de valor complementario en origen por lo que su rentabilidad es baja y poco distribuida.

El segundo se refiere al sistema tecnológico. La falta de reconocimiento profundo y valorización de la materia prima (pino ponderosa) que se produce en esta región, hace que no se generen plusvalías (diseño-desarrollo-producción-certificación y normativa) y por tanto, una producción de gran superficie genera baja rentabilidad, con los consecuentes detrimentos en la dinámica de la economía de la zona.

El tercero es el sistema socio productivo y se refiere a la economía monopolizada por la actividad turística de tipo estacional, que genera trabajo insuficiente y discontinuo en la región, restringido a períodos de temporada alta turística, dejando un vacío importante en el empleo anual, especialmente en épocas intermedias y bajas. La posibilidad de dinamizar el sector forestal en Bariloche permitirá una renta anual constante, una diversificación en el empleo y la generación de trabajo autónomo con baja inversión de capital inicial permitiendo la inclusión, en la cadena de valor del sector forestal, de los grupos económicos más vulnerables -micro-emprendimientos, cooperativas, talleres productivos, etc.

\section{Conclusiones no finales.}

Hasta el momento la experiencia en Bariloche ha generado una Red Interactoral Productiva representada por la Cooperativa de Trabajo Laburar, el Taller de Oficios de la Escuela Angelelli, el Taller San José Obrero, sumándose a la Red otros actores que han colaborado con el financiamiento tales como el Instituto Municipal de Tierra y Vivienda para el Hábitat Social, la Subsecretaría de Desarrollo Local, la Subsecretaría de Recursos Forestales de la Provincia; también forman parte de esta experiencia instituciones de investigación como el INTA- EEA Bariloche, CIECS-CONICET-UNC (en cual los autores de este trabajo tiene sede); participan a su vez de este proceso tecnológico otros actores de preciado interés como comerciantes, aserraderos locales y la Junta Vecinal del Barrio 96 Viviendas, donde se está construyendo actualmente un Salón de Usos Múltiples, que de alguna manera se constituye en la materialización de la experiencia.

La experiencia de co-construcción sostuvo en el inicio, a partir de la conformación de la Red, una serie de Encuentros Productivos a lo largo de los cuales se fue diseñando y desarrollando la tecnología constructiva en madera de pino ponderosa con la participación de todos los actores involucrados en dicha Red. 


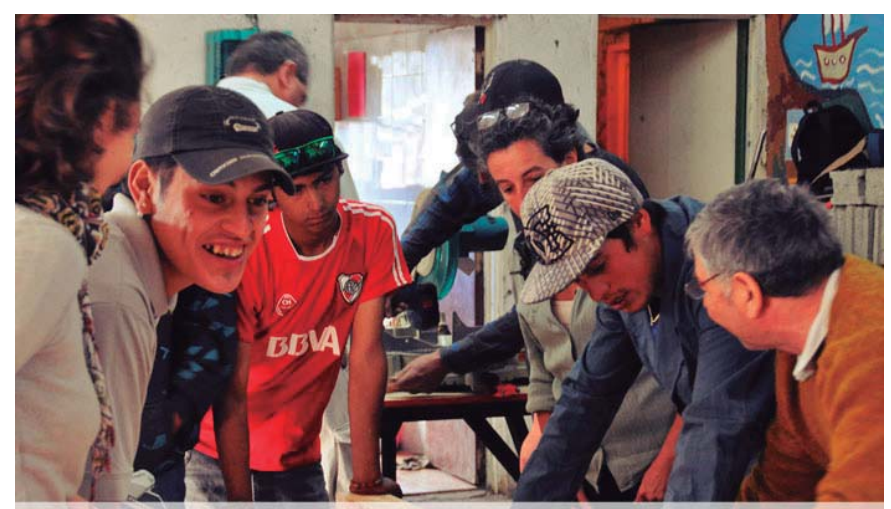

Figura 1. Encuentro Productivo en Taller San José Obrero.

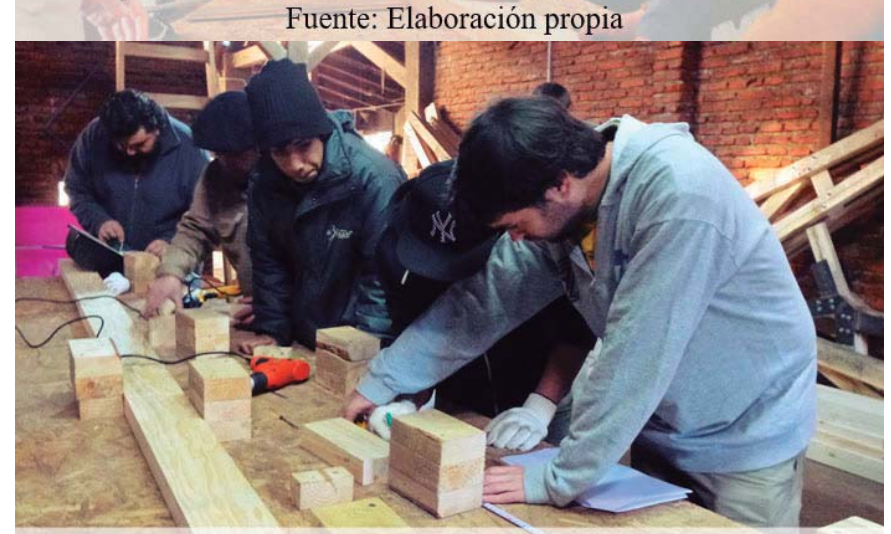

Figura 2. Encuentro Productivo en Taller San José Obrero.

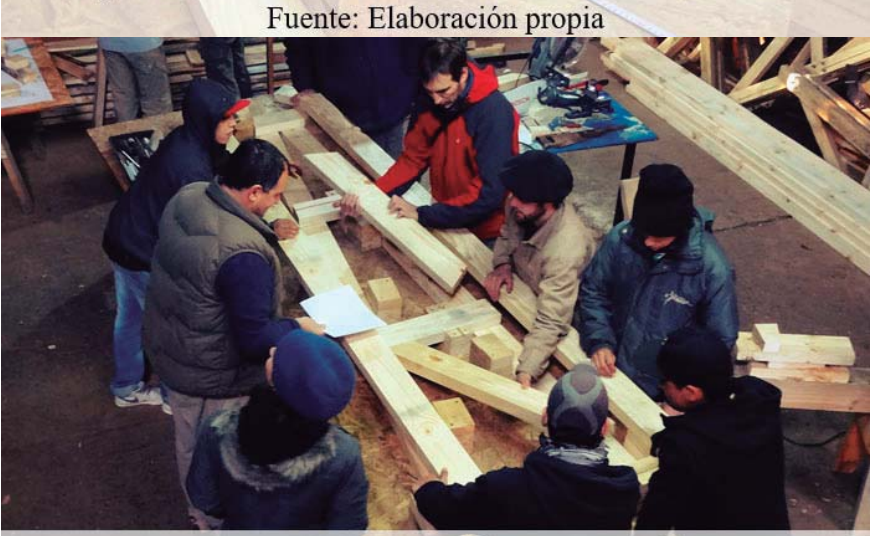

Figura 3. Encuentro Productivo en Taller San José Obrero. Fuente: Elaboración propia

Estos talleres de producción colectiva permitieron realizar los primeros componentes constructivos prefabricados y estandarizados constituyéndose en simulaciones, prototipos y mini-series de producción a partir de los cuales se fue ajustando el producto hasta alcanzar su última propuesta, en el marco de la integración de saberes diversos y complementarios.

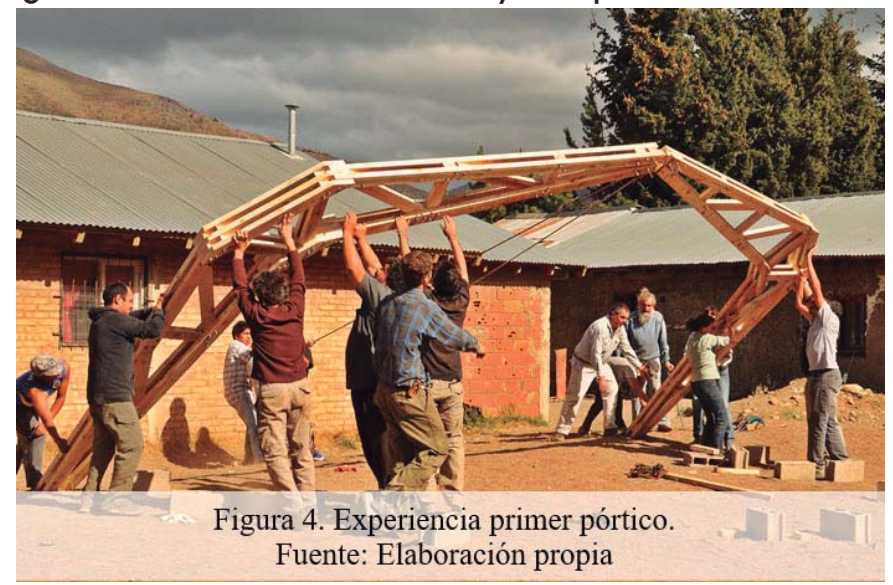




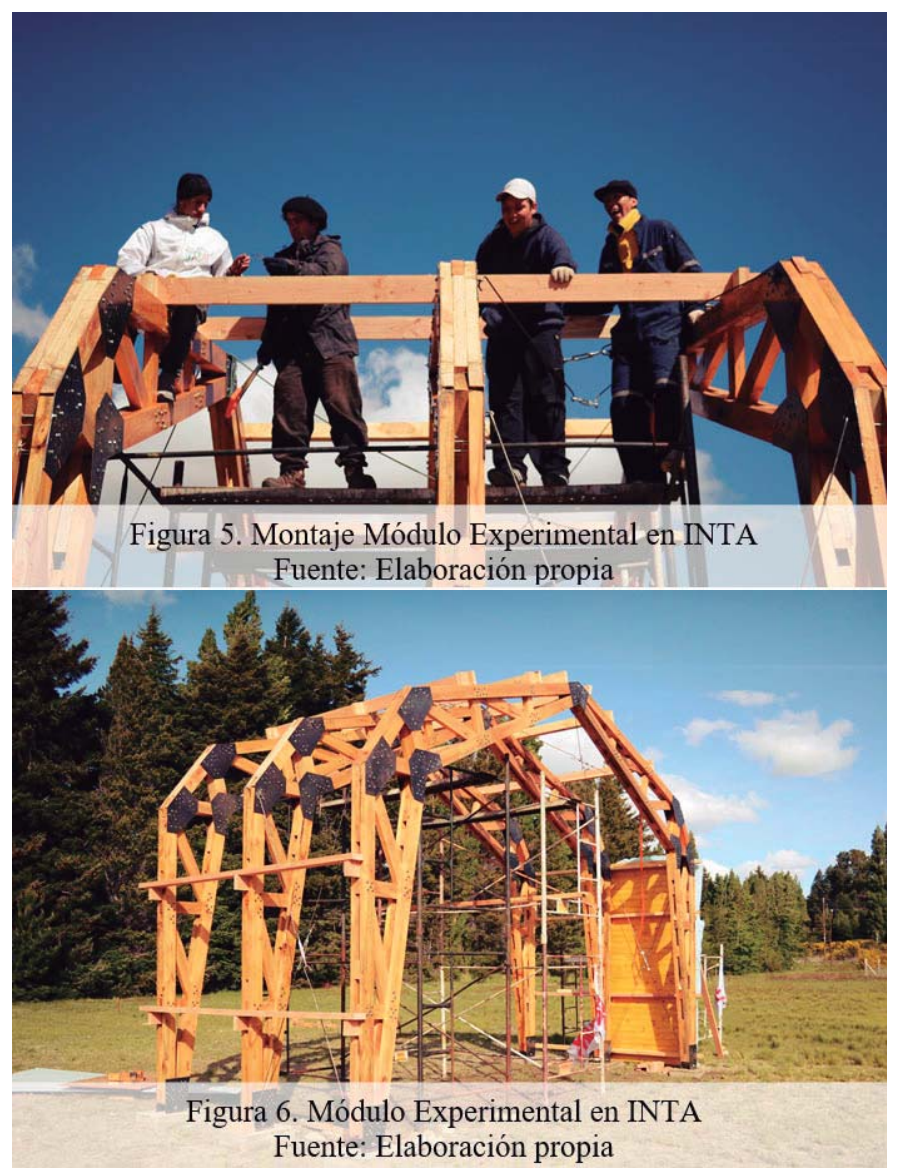

Entre medio de este accionar técnico, de talleres experimentales de diseño, desarrollo y producción, se fueron realizando otro tipo de reuniones signadas por la toma de decisiones en conjunto, generando legítimos espacios de comunicación, información y participación.

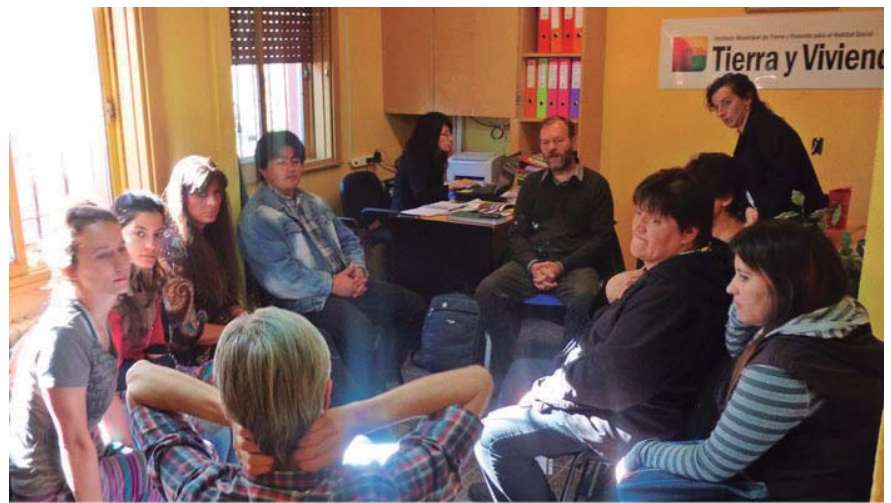

Figura 7. Reunión inter-actoral en Instituto de Tierra y Vivienda

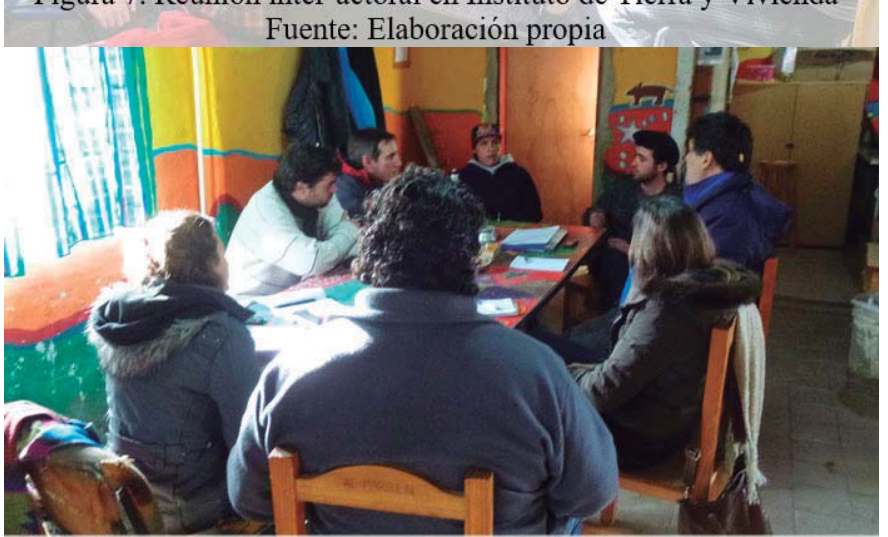

Figura 8. Reunión inter-actoral en Taller Angelelli Fuente: Elaboración propia 


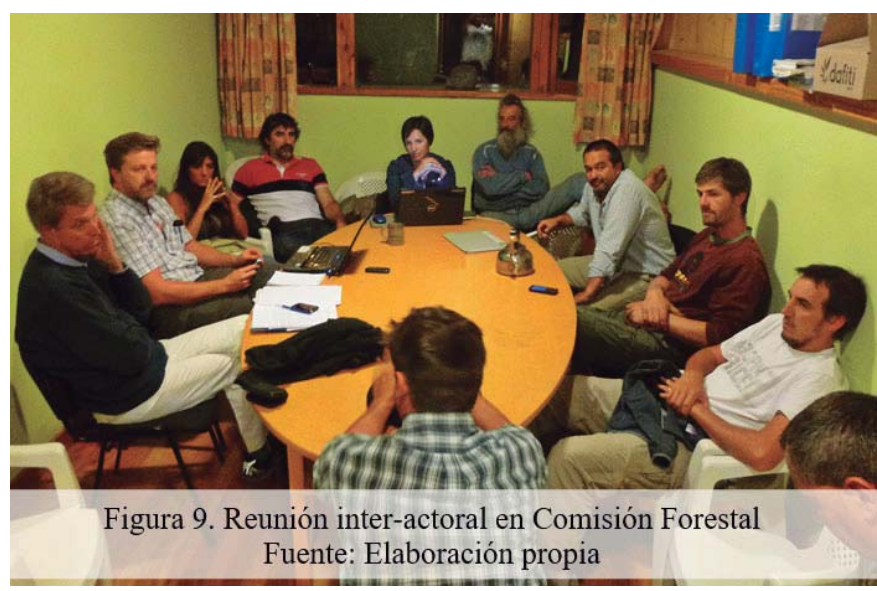

El Proceso Productivo Interactoral e Inclusivo en Bariloche se ha ido desenvolviendo como una manera de generar una respuesta diferenciada para el desarrollo de tecnología, que asuma un involucramiento de los sectores más vulnerables de la comunidad, que suponga una construcción cognitiva mixta de saberes académicos y vivenciales, que mantenga en estado democrático los procesos de toma de decisiones a través de una comunicación abierta y participativa, que haga uso de recursos locales renovables en sintonía con un buen manejo de los mismos, que promueva la integración socio productiva de la región de una manera complementaria y no competitiva, que pueda ser propiciada por el Estado como una política de cambio y transformación para generar una economía solidaria, cooperativa y asociativa.

Solo bajo estas condiciones será posible encontrar plusvalías en la producción forestal de la región. La cadena de valor debe asumirse entonces no necesariamente por plusvalías en torno a la renta específicamente sino a partir de un desarrollo tecnológico (producto-proceso-gestión) que garantice inclusión socio productiva bien entendida.

En la actualidad se está finalizando el Salón de Usos Múltiples en el barrio 96 Viviendas en San Carlos de Bariloche bajo esta convicción colectiva.

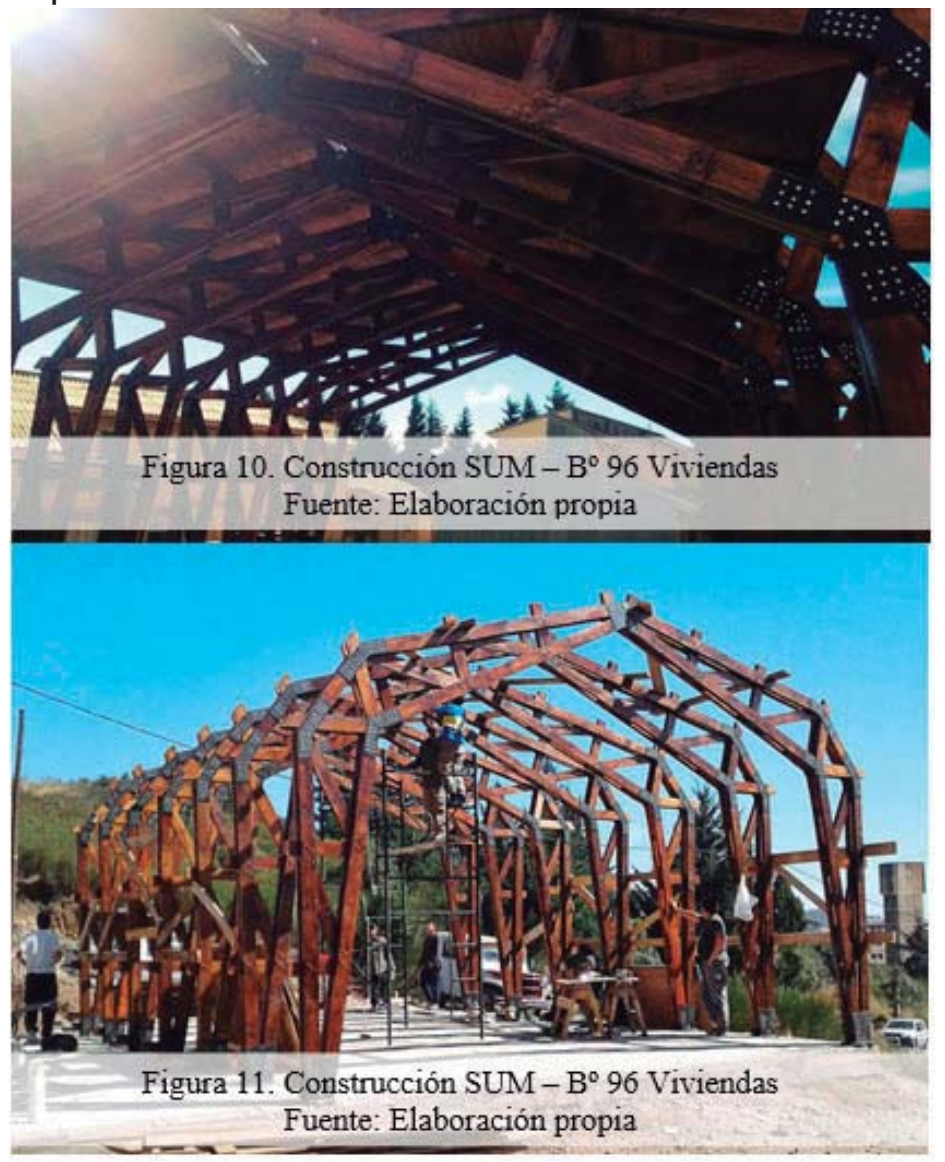




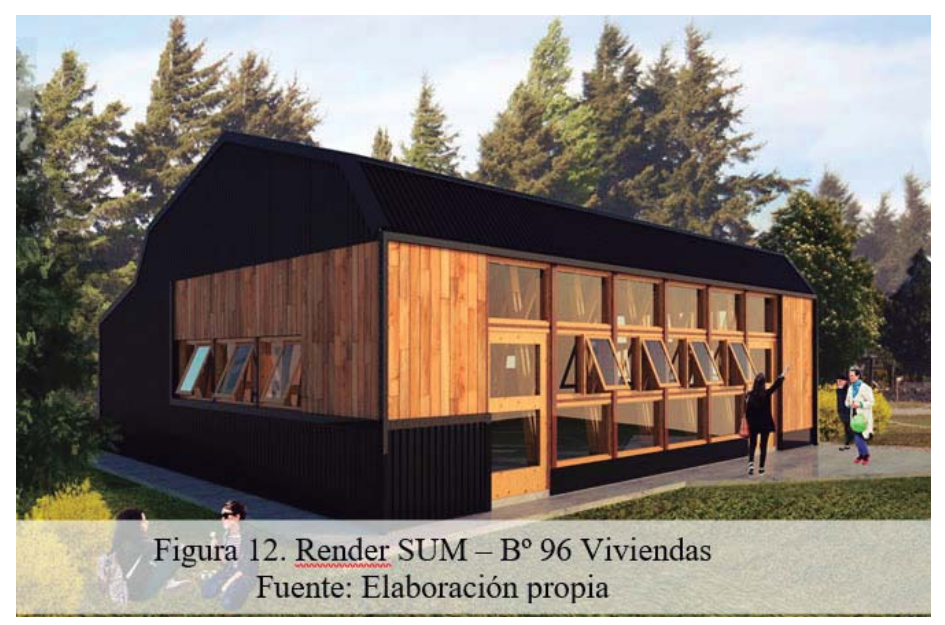

\section{Referencias}

DAGNINO, Renato. Tecnología Social. Uma estrategia para o desenvolvimento. CIP Brasil. Rio de Janeiro. 2004

FEYERABEND, Paul. Tratado contra el método. (Orig. 1975). México DF. Siglo XXI. 2010:29-166

PEYLOUBET, Paula. Animarse a habitar. Argentina. Buenos Aires. Ed. Diseño. 2017

THOMAS, Hernán. Tecnologías para Inclusao Social e Politicas Públicas no América Latina en Tecnologías Sociaes. Caminhos para a sustentabilidade. Red de Tecnología Social. Brasilia.2009 